\title{
Limb preservation with suprafascial and thin perforator flaps: salvaging osteomyelitis, Charcot collapse and critical limb ischemia
}

\author{
Shawn Diamond ${ }^{1}$, Andres F. Doval ${ }^{1}$, Benjamin Scott ${ }^{1}$, Matthew L. Iorio ${ }^{2}$ \\ 'Division of Plastic and Reconstructive Surgery, Beth Israel Deaconess Medical Center, Harvard Medical School, Boston, MA 02215, USA. \\ ${ }^{2}$ Division of Plastic and Reconstructive Surgery, University of Colorado Anschutz Medical Center, Aurora, CO 80045, USA.
}

Correspondence to: Dr. Matthew L. Iorio, Division of Plastic and Reconstructive Surgery, University of Colorado Anschutz Medical Center, 12631 E. 17th Ave, C309 (Room 6414), Aurora, CO 80045, USA. E-mail: matt.iorio@ucdenver.edu

How to cite this article: Diamond S, Doval AF, Scott B, lorio ML. Limb preservation with suprafascial and thin perforator flaps: salvaging osteomyelitis, Charcot collapse and critical limb ischemia. Plast Aesthet Res 2019;6:20. http://dx.doi.org/10.20517/2347-9264.2019.26

Received: 3 Jun 2019 First Decision: 11 Jul 2019 Revised: 17 Jul 2019 Accepted: 20 Jul 2019 Published: 12 Aug 2019

Science Editor: Raúl González-García Copy Editor: Cai-Hong Wang Production Editor: Tian Zhang

\begin{abstract}
Aim: There are limited reports in the United States demonstrating outcomes of primarily thinned fasciocutaneous flaps in the setting of critical limb ischemia, Charcot collapse and osteomyelitis. We hope to determine patient and flap related outcomes in advanced lower extremity disease.
\end{abstract}

Methods: The authors conducted a retrospective review of fasciocutaneous free flaps of variable thickness for lower extremity salvage. Osteomyelitis and non-osteomyelitis patients were compared according to our primary outcome measures: functional ambulation, bone healing and complications to flap and patient. Subgroups with critical limb ischemia, Charcot collapse and diabetic foot were analyzed separately.

Results: Fifty-nine patients underwent free flap reconstruction: osteomyelitis $(n=20,34 \%)$, Charcot collapse ( $n=22$, $37 \%)$, and/or critical limb ischemia $(n=12,20 \%)$. All patients underwent anterolateral thigh flaps tailored for defectspecific thicknesses: 17 superthin, 25 suprafascial, 17 subfascial. There were no significant differences between groups in terms of partial and complete flap loss $(P=1.000$ and $P=0.108)$. Ninety-one percent of patients were ambulatory at follow up. Eighty-five percent of individuals with osteomyelitis cleared their infection demonstrating radiographic bone healing. Two patients developed recalcitrant deep space infections ultimately requiring amputation. Subgroup analysis did not show any differences in flap related complications within the diabetic Charcot population. In multivariate regression, preoperative revascularization was independently associated with failure of limb salvage.

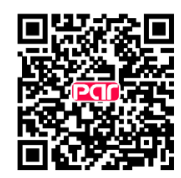


Conclusion: Primarily thinned perforator flaps performed well in the setting of lower extremity limb salvage, critical limb ischemia, osteomyelitis, and the Charcot foot - expanding their role in the armamentarium for lower extremity care.

Keywords: Perforator flap, diabetic foot, limb salvage

\section{INTRODUCTION}

Free microvascular tissue transfer in combination with aggressive debridement, targeted antimicrobial therapy, optimization of distal perfusion and boney stabilization remains a powerful tool to heal lower extremity wounds with osteomyelitis - restoring functional ambulation ${ }^{[1-3]}$. Godina along with Mathes described the role of muscle-flap coverage for high-energy wounds with infected bone almost 40 years ago. They achieved an $89 \%$ infection clearance rate ${ }^{[4]}$. Numerous authors have demonstrated similar results ${ }^{[1-7]}$. Lower extremity salvage in the setting of high-energy trauma, critical limb ischemia and the diabetic foot often includes management of denuded and dysvascular bone with variable degrees of osteomyelitis ranging from superficial contamination, to deeper medullary involvement, from localized to diffuse infections described by the four-tiered Cierny-Mader classification ${ }^{[8,9]}$. Traditionally muscle-bearing flaps were used to create a local tissue environment conducive to healing and fill-in tissue dead-space. Muscle has been thought of as more effective than fasciocutaneous flaps in overcoming bacterial colonization and infection due to improved oxygen delivery and restoration of wound bed perfusion ${ }^{[2,3,6,10]}$. However this has been refuted over the past decade by a number of authors ${ }^{[3,5,7,11]}$.

Over time, a deeper understanding of perforasome anatomy ${ }^{[12]}$, microsurgical technical refinements ${ }^{[13-16]}$, perioperative protocols and improved instrumentation has empowered reconstructive surgeons to reliably utilize skin-only and fasciocutaneous flaps for coverage of lower extremity defects ${ }^{[17]}$. Nonetheless, challenges remain as traditional perforator-based flap thickness can interfere with post-operative function, particularly in the lower extremity, wherein bulky, thick, flaps can interfere with footwear, contour across joints, irregular weight bearing surface and can lead to flap breakdown ${ }^{[2,13,14]}$. Technical refinements in anterolateral thigh (ALT) harvest offer reliable methods to achieve thinner flaps, minimizing debulking procedures, improving contour and decreasing donor morbidity.

Recent reports consistently demonstrate that elevation of the ALT flap in different planes allow for the possibility of safe, consistent, and definitive distal extremity reconstruction in a single stage ${ }^{[15,18,19]}$. However, limited data exists for successful limb salvage with use of thin fasciocutaneous flaps in the setting of osteomyelitis, limb ischemia and the Charcot foot. Our goal is to describe the routine use of the primarily thinned ALT flap in varying thicknesses for lower limb salvage surgery, and to assess outcomes in patients at high risk for failure.

\section{METHODS}

\section{Patient data}

All lower extremity free flaps performed at a single, Level 1 medical center were entered into a prospectively maintained registry including patient demographic information, clinical history, radiographic imaging, procedural data, operative reports, postoperative care and long-term complications across 116 unique variables. A REDCap database was utilized as a secure web-based application for data maintenance. A trained member of the research team uploads data once monthly. Follow up radiographic reports and clinic visits are specifically analyzed to identify limb salvage failure, nonunion, malunion, osteomyelitis, flap failure, wound recurrence, patient ambulation, use of assistive devices, patient disposition and rates of amputation. The database is maintained via institutional review board approval. 

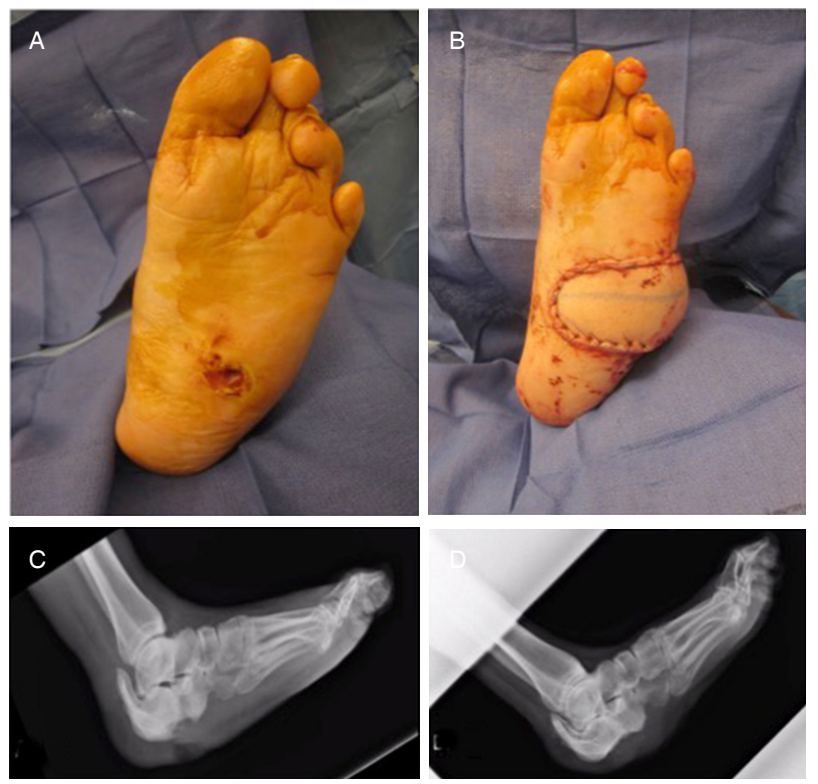

Figure 1. Superficial osteomyelitis managed with suprafascial anterolateral thigh. A patient with Cierny-Mader Class 2 ostomyelitis of the calcaneus. A: A preoperative photo; B: immediate post flap phot; C: pre flap radiography with osteomyelitis; D: six-month follow up photo with clearance of osteomyelitis. The patient was weight bearing at time of follow up

For the purposes of this study, the database was queried in June 2018 for cases performed from January 2015 to December 2017. We excluded muscle flaps and skin-only or fasciocutaneous flaps other than ALT's. Of 84 patients who underwent lower extremity free-tissue with ALT flaps, we excluded 25 individuals without high risk factors. This left 59 patients selected for at least one of the following features: osteomyelitis, Charcot collapse, and critical limb ischemia.

For each patient, relevant demographic information, comorbidities, presence of peripheral vascular disease, revascularization, antibiotic use, anticoagulant use, wound etiology, pre-operative imaging, anatomical wound location, skeletal fixation, flap thickness, operative characteristics, complications and follow-up were reviewed.

Osteomyelitis was defined as tissue-proven boney infection via histological analysis and bony tissue culture obtained at the time of flap coverage in the case of single-staged reconstructions and or prior to reconstruction from bone biopsy. Clinical, radiographic, microbiological information was gathered.

Patients were separated based on the Cierny-Mader classification system defining the depth as well as diffusion of osteomyelitis ${ }^{[8,9,20]}$. Patients with Charcot collapse, critical limb ischemia (defined by vascular imaging proven: single vessel run-off, multi-level or multivessel arterial disease) and diabetes mellitus were separated into a subgroup for analysis of their unique pathophysiology.

\section{Reconstructive technique}

Patients were separated for analysis into groups based on flap thickness: periscarpal (superthin), suprafascial and subfascial (thick). During the period of study, no muscle-flaps were utilized. We relied uniformly on skin-only and fasciocutaneous flaps. Flap thickness was tailored to match defect surface contour and volume of dead space.

Figures 1-3 demonstrate case based examples of our reconstructive technique. ALT flap thickness for each case was determined by defect thickness, need to fill deadspace and correlates with Cierny classification of Osteomyelitis. 

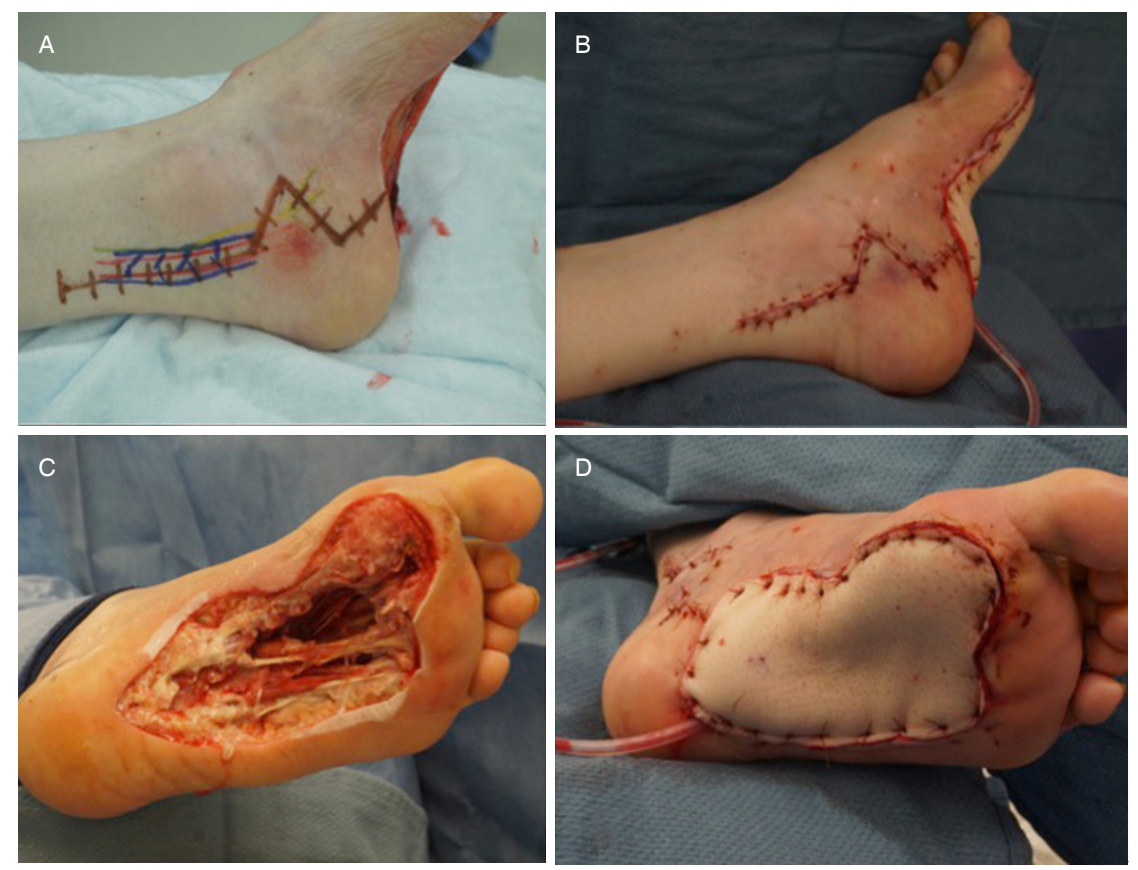

Figure 2. Deep Space Infection and Charcot Foot treated with Subfascial anterolateral thigh (ALT). A patient with plantar weight bearing soft tissue loss after deep space diabetic foot infection. A: Preoperative lateral view showing Bruner incision for tarsal tunnel release, vessel harvest and tibial neurolysis; B: lateral clinical photography after inset and closure of Bruner incision with mid-foot plantar arch contouring; C: preoperative view of the plantar surface; D: after subfascial ALT for coverage and lateral femoral cutaneous nerve coaptation to plantar branch of the tibial nerve. The crural fascia was inset well beyond the skin incision margin. The patient was successfully weight bearing 11 weeks post-operatively

Our technique for elevation in the desired plane has been previously described ${ }^{[15,16]}$. Superthin flaps were defined as those elevated at the superficial scarpal fascia within the subcutaneous fat. Suprafascial flaps were defined as flaps elevated just above the crural fascia and subfascial flaps were those elevated below the crural fascia and/or deep muscular fascia ${ }^{[2]}$. Defects with bone-loss requiring spacer placement and or bone grafting for management of later stage III, IV Cierny-Mader osteomyelitis often required thicker flaps to fill-in dead space. As such, subfascial ALT flaps were harvested to assist filling dead-space and or to contour deeper defects. However, we preferentially utilized a superthin elevation for reconstruction of weight bearing surfaces along the heal, mid-foot, dorsal-foot and ankle region. Earlier stage CiernyMader Osteomyelitis being cortical, focal medullary involvement resulted in superficial boney defects often amenable to coverage with super-thin flaps.

The major tenets of lower extremity salvage were regarded as appropriate debridement to perfused tissue, preservation of vital structure, muscle, nerve and tendon along with isolation and control of major vascular inflow. Wounds amenable to local tissue reconstruction with advancement flaps, skin-graft, regional pedicle flaps, freestyle propeller flaps were utilized when-able but were excluded from this study.

During free tissue transfer, we preferentially performed end-end anastomosis in patients with adequate runoff and normal vascular supply. However, in patients with peripheral vascular disease, single-vessel runoff, multi-vessel or multi-level flow limiting lesions, end to side anastomosis was performed to maintain in-line perfusion distal to the reconstruction. Venous outflow was preferentially based on the deep venous system with emphasis on vessel quality, size-match, lack of back-bleeding, avoidance of venous hypertension over absolute number of venous anastomosis.

An enhanced recovery protocol was utilized for the majority of our patients including the use of regional anesthetic block achieved via continuous peripheral nerve catheter placed in the popliteal region 

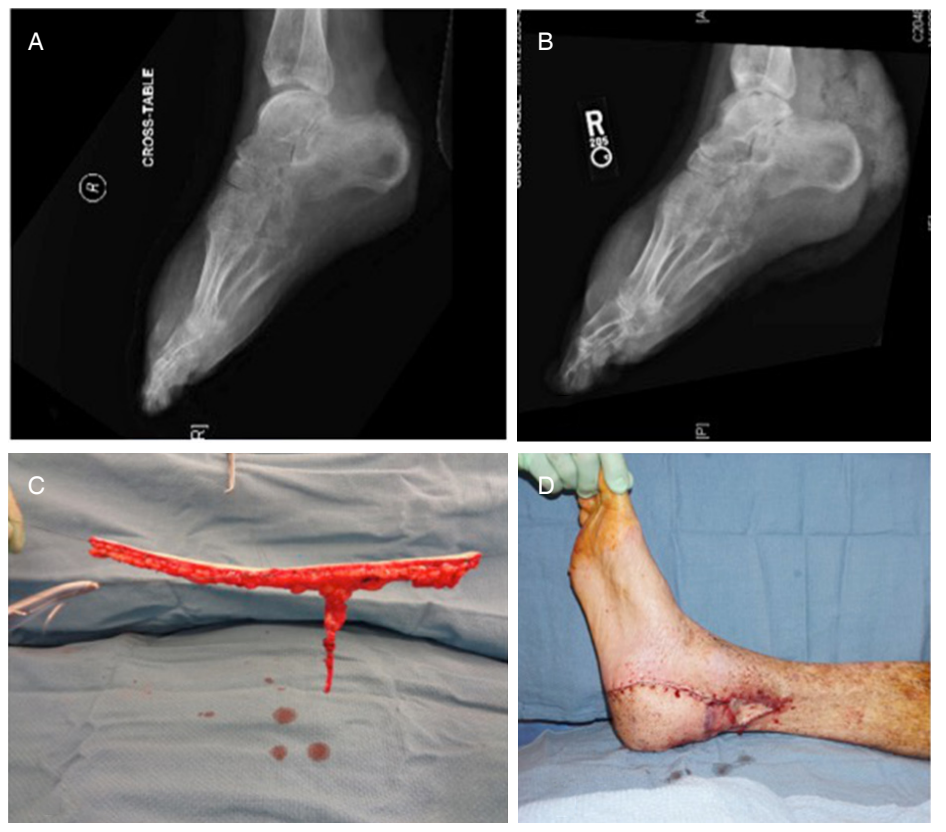

Figure 3. A case of Calcaneal Osteomyelitis treated with superthin anterolateral thigh (ALT) flap. A patient with Cierny-Mader class 3 osteomyelitis with calcaneal and proximal mid-foot erosions seen on (A) preoperative radiography. This required boney debridement and soft tissue coverage with superthin ALT in single-stage; (B) shows postoperative radiography with clearance of osteomyelitis; (C) demonstrating superthin (periscarpal) ALT; (D) after final inset and small skin graft for coverage of the vascular pedicle. This resulted in full-ambulation in normal shoe gear

targeting the sciatic, tibial and peroneal nerves when appropriate. This achieved decreased rates of postoperative narcotic use and shorter post-anesthesia unit stays ${ }^{[21]}$. Additionally an early limb dependency program $^{[22]}$ helped patients dangle early in their post-operative course expediting hospital stay, discharge to rehabilitation facilities, and return toward functional ambulation.

\section{Outcome measures}

Outcomes pertaining to flap specific morbidity such as partial flap loss, microvascular collapse, vessel thrombosis, site infection and dehiscence were analyzed in addition to systemic complications.

With regard to osteomyelitis, discontinuation of antibiotic, achievement of boney union, return to weight bearing, exchange of external for internal hardware and radiography were analyzed. Return to functional ambulation, weight bearing and avoidance of amputation were compared across all groups.

\section{Statistical analysis}

Descriptive statistics were utilized to compare patient demographic information in regard to number, frequency, mean and standard deviation. Student $t$-test for continuous data and Fischer's exact test for categorical data were used for univariate analysis to determine significant differences in wound and flap characteristics along with donor site and flap complications between groups of patients. Those variables achieving significance $P$-value $<0.05$ were entered into a multivariable regression model to identify independent risk factors associated with limb loss and osteomyelitis recurrence. Statistical significance was defined as a $P$-value $<0.05$. The analysis was performed using IBM SPSS software by members of our research group within the institution (Version 3.154).

\section{RESULTS}

A total of 59 ALT flaps were performed from January 2015 to December 2017 for lower extremity salvage reconstruction. The mean follow-up time of our population was 13.8 months (2.24-39.2 months). Flaps 
Table 1. Patient characteristics and univariate comparison based on the presence of osteomyelitis

\begin{tabular}{|c|c|c|c|}
\hline & Osteomyelitis $n=20$ & No osteomyelitis $n=39$ & P-value \\
\hline Age & $51.95 \pm 13.65$ & $52.3 \pm 13.44$ & 0.918 \\
\hline $\operatorname{Sex}(\%)$ & & & 0.192 \\
\hline Male & $18(90)$ & $29(74.4)$ & \\
\hline Female & $2(10)$ & $10(25.6)$ & \\
\hline BMI & $31.53 \pm 6.80$ & $28.10 \pm 4.71$ & 0.026 \\
\hline Smoking history (\%) & $6(30.0)$ & $17(43.6)$ & 0.311 \\
\hline Hypertension (\%) & $9(45.0)$ & $10(25.6)$ & 0.132 \\
\hline Diabetes (\%) & $9(45.0)$ & $13(33.3)$ & 0.380 \\
\hline Coronary artery disease (\%) & $5(25.0)$ & $3(7.7)$ & 0.106 \\
\hline Peripheral artery disease (\%) & $6(30.0)$ & $9(23.1)$ & 0.563 \\
\hline Renal disease (\%) & $1(5.0)$ & $3(7.07)$ & 1.000 \\
\hline Malnourished (Albumin < $3.5 \mathrm{~g} / \mathrm{dL}$ ) (\%) & $6(30.0)$ & $5(12.8)$ & 0.159 \\
\hline Multiple comorbidities (\%) & $10(50.0)$ & 23 59.0) & 0.511 \\
\hline Preoperative antithrombotic use (\%) & $20(100.0)$ & $38(97.4)$ & 1.000 \\
\hline Preoperative antiplatelet use (\%) & $12(60.0)$ & $27(69.2)$ & 0.478 \\
\hline Chemotherapy (\%) & $1(5.0)$ & $3(7.7)$ & 1.000 \\
\hline Radiation (\%) & $1(5.0)$ & $3(7.7)$ & 1.000 \\
\hline Prior revascularization (open or endovascular) (\%) & $6(30.0)$ & $3(7.7)$ & 0.050 \\
\hline Vascular imaging (\%) & $13(65.0)$ & $22(56.4)$ & 0.525 \\
\hline Prior amputation level (\%) & $2(10.0)$ & $0(0.0)$ & 0.111 \\
\hline
\end{tabular}

Table 2. Wound, flap and anastomotic characteristics with univariate comparison between groups of patients based on the presence of osteomyelitis

\begin{tabular}{|c|c|c|c|c|c|}
\hline & No. & $\%$ & Osteomyelitis $(n=20)$ & No osteomyelitis $(n=39)$ & $P$-value \\
\hline \multicolumn{6}{|l|}{ Type of wound (\%) } \\
\hline Traumatic & 30 & 50.8 & $7(35.0)$ & $23(59.0)$ & 0.081 \\
\hline Malignant & 5 & 8.5 & $0(0.0)$ & $5(12.8)$ & 0.156 \\
\hline Chronic (> 90-day) & 24 & 40.7 & $13(65.0)$ & $11(28.2)$ & 0.006 \\
\hline \multicolumn{6}{|l|}{ Wound location } \\
\hline Foot & 24 & 40.7 & $8(40.0)$ & $16(41.0)$ & 0.939 \\
\hline Ankle & 14 & 23.7 & $5(25.0)$ & $9(23.1)$ & 1.000 \\
\hline Calf & 13 & 22.0 & $4(20.0)$ & $9(23.1)$ & 1.000 \\
\hline Shin & 3 & 5.1 & $1(5.0)$ & $2(5.1)$ & 1.000 \\
\hline Knee & 4 & 6.8 & $2(10.0)$ & $2(5.1)$ & 0.598 \\
\hline Thigh & 1 & 1.7 & $0(0.0)$ & $1(2.55)$ & 0.544 \\
\hline \multicolumn{6}{|l|}{ Flap type } \\
\hline Superthin & 17 & 28.8 & $8(40.0)$ & $9(23.1)$ & 0.174 \\
\hline Suprafascial & 25 & 42.4 & $8(40.0)$ & $17(43.6)$ & 0.792 \\
\hline Subfascial & 17 & 28.8 & $4(20.0)$ & $13(33.3)$ & 0.284 \\
\hline \multicolumn{6}{|l|}{ Anastomosis } \\
\hline End to End & 42 & 76.4 & $15(75.0)$ & 27 (77.1) & 1.000 \\
\hline End to Side & 13 & 23.6 & $5(25.0)$ & $8(22.9)$ & 1.000 \\
\hline
\end{tabular}

were elevated in three major planes: superthin (17 patients, $29 \%$ ), suprafascial (25 patients, $42 \%$ ) and subfascial (17 patients, 29\%). Twenty patients (34\%) had tissue-proven osteomyelitis. When separated into groups based on the presence of osteomyelitis, patients were well-matched across 116 variables. Select comparisons are shown in Table 1. Individuals with osteomyelitis had higher average BMI $(P=0.03)$ and a greater incidence of previous vascular interventions $(P=0.05)$.

Table 2 describes wound characteristics and locations. Traumatic injury was the most common type of wound etiology in both osteomyelitis and non-osteomyelitis groups $(51 \%)$ followed by chronic wound and malignant wounds ( $40 \%$ and $9 \%$, respectively). Chronic wounds were defined as an established wound despite attempts at local or surgical wound care, offloading, and medical comorbidity management past 90days. The foot and ankle were the most common recipient site for the ALT flaps. The distributions of ALT thickness type across osteomyelitis as well as non-osteomyelitis groups were comparable. 
Table 3. Flap complication and reported Limb salvage rates

\begin{tabular}{|c|c|c|c|c|c|}
\hline & Total N N.59 = 59 & Osteomyelitis N.2ON = 20 & 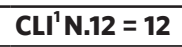 & Charcot N.22 & $P$-value ${ }^{2}$ \\
\hline Ambulatory & $54(91 \%)$ & $17(85 \%)$ & $10(83 \%)$ & $20(90 \%)$ & 0.110 \\
\hline Non-ambulatory & $5(9 \%)$ & $3(15 \%)$ & $2(17 \%)$ & $2(9.1 \%)$ & 0.217 \\
\hline Amputation & $2(3.4 \%)$ & $2(10 \%)$ & $2(17 \%)$ & $2(9.1 \%)$ & 0.252 \\
\hline Amputation free & $57(96.6 \%)$ & $18(90 \%)$ & $10(83 \%)$ & $20(90 \%)$ & \\
\hline Osteomyelitis clearance & & $18(90 \%)$ & $10(83 \%)$ & $18(81 \%)$ & \\
\hline Flap complication & $18(30 \%)$ & $8(40 \%)$ & $5(42 \%)$ & $7(31.8 \%)$ & 0.260 \\
\hline Complete flap loss & $4(6.8 \%)$ & $1(5 \%)$ & $0(0 \%)$ & $0(0 \%)$ & 0.699 \\
\hline Partial flap loss & $3(5.1 \%)$ & $1(5 \%)$ & $2(17 \%)$ & $2(9.1 \%)$ & 0.983 \\
\hline Flap infection & $4(6.8 \%)$ & $2(10 \%)$ & $1(8.3 \%)$ & $1(4.5 \%)$ & 0.598 \\
\hline Dehiscence & $6(10.2 \%)$ & $1(5 \%)$ & $1(8.3 \%)$ & $4(18.2 \%)$ & 0.653 \\
\hline Flap revision & $9(15 \%)$ & $1(5 \%)$ & $1(8.3 \%)$ & $0(0 \%)$ & 0.841 \\
\hline
\end{tabular}

${ }^{1} \mathrm{CLI}$ : critical limb ischemia, defined as patients with at least one of the following: single-vessel runoff, severe peripheral vascular disease, multi-vessel arterial disease, multi-level arterial disease; ${ }^{2} P$-values reported after univariate comparison of patients with Osteomyelitis to those without across all outcomes

Overall donor site complication rates were as follows: seroma, $5 \%(n=3)$; neuropathy, $5 \%(n=3)$; contour irregularity, $3.4 \%(n=2)$; and site infection, $3.8 \%(n=2)$. No differences in donor site complication rates were noted between groups. Flap complication rates including complete flap loss, partial flap loss, wound dehiscence and flap infection were $6.8 \%, 5.1 \%, 10.2 \%$, and $6.8 \%$, respectively. Again, no significant differences between groups were noted. Table 3 demonstrates complication rates across high-risk individuals harboring critical limb ischemia, Charcot foot, and osteomyelitis. Eight flap revisions were needed, none of which were related to an osteomyelitis recurrence.

Sub-group analysis in the diabetic population revealed no cases of complete flap loss, two cases of partial flap loss, four cases of wound dehiscence and one of flap infection, complication rates of $0.0 \%, 9.1 \%, 18.2 \%$ and $4.5 \%$, respectively [Table 3]. No differences between the osteomyelitis and non-osteomyelitis groups within the diabetic population were noted. Our sub-group analysis demonstrated comparable outcomes amongst a group of diabetic patients with osteomyelitis and Charcot foot.

Table 4 describes variegations in the osteomyelitis group and associated salvage rates. Cierny-Mader osteomyelitis class, flap type and presence of comorbidity did not significantly alter osteomyelitis clearance $(P>0.05)$ for each univariate comparison.

A multivariable regression was performed after stepwise entry of variables associated with limb-loss and amputation with $(P<0.1)$. Preoperative revascularization was independently associated with limb loss OR $6.1(P<0.05)$. Osteomyelitis, Charcot foot, diabetes, the presence of critical limb ischemia, and flap elevation plane were not in and of themselves independently associated with limb loss.

\section{DISCUSSION}

In this study of lower extremity free tissue transfers with ALT flaps, we compared complication rates and outcomes across three elevation planes in settings of osteomyelitis, charcot collapse and critical limb ischemia. We did not find any significant differences between the groups using either one of the three planes of elevation in terms of major complication as flap revision, flap infection; wound dehiscence, partial and complete flap loss. Important to note, the distribution of flap types between groups harboring osteomyelitis was comparable (superthin: $P=0.174$; suprafascial: $P=0.792$; and subfascial: $P=0.284$ ). Interestingly, there were no differences between major flap complications within the diabetic population and Charcot foot. These findings further support the use of skin-only and fasciocutaneous flaps in the setting of osteomyelitis. We demonstrate that thin flaps can assist in boney healing and clearance of infection despite a lower metabolic demand compared to muscle flaps. 
Table 4. Characteristics of 20 patients who underwent perforator based flaps for treatment of lower extremity osteomyelitis

\begin{tabular}{|c|c|c|c|}
\hline & No. & (\%) & Clearance $^{a}$ \\
\hline \multicolumn{4}{|l|}{ Soft tissue defect location } \\
\hline Calf/Knee & 7 & 35 & $7(100 \%)$ \\
\hline Ankle & 5 & 25 & $4(80 \%)$ \\
\hline Foot & 8 & 40 & $7(87.5 \%)$ \\
\hline \multicolumn{4}{|l|}{ Bone involved } \\
\hline Tibia & 5 & 25 & $5(100 \%)$ \\
\hline Fibula & 2 & 10 & $2(100 \%)$ \\
\hline Calcaneus & 5 & 25 & $4(80 \%)$ \\
\hline Ankle mortis/carpus & 6 & 30 & $6(100 \%)$ \\
\hline Metatarsal/phalangeal & 2 & 10 & $1(50 \%)$ \\
\hline Tissue based diagnosis & 20 & 100 & \\
\hline \multicolumn{4}{|l|}{ Cierney-Mader classification } \\
\hline I - Superficial & 12 & 60 & $12(100 \%)$ \\
\hline II - Medullary & 3 & 15 & $2(67 \%)$ \\
\hline III - Isolated (Sequestrum) & 1 & 5 & $1(100 \%)$ \\
\hline IV - Diffuse & 4 & 20 & $1(75 \%)$ \\
\hline Hardware present and kept in place & $(4 / 4)$ & 100 & $4(100 \%)$ \\
\hline External fixator present & 2 & 10 & $2(100 \%)$ \\
\hline \multicolumn{4}{|l|}{ Microorganism } \\
\hline Staph epidermidus, coagulase negative staph. MSSA & 13 & 65 & $12(92 \%)$ \\
\hline MRSA & 1 & 5 & $1(100 \%)$ \\
\hline Enterobacter & 2 & 10 & $2(100 \%)$ \\
\hline Streptococcal & 1 & 5 & $1(100 \%)$ \\
\hline Corynebacterium & 1 & 5 & $1(100 \%)$ \\
\hline Proteus sp. & 1 & 5 & $0(0 \%)$ \\
\hline Stenotrophomonas & 1 & 5 & $1(100 \%)$ \\
\hline \multicolumn{4}{|l|}{ Flap thickness } \\
\hline Subfascial (Thick) & 4 & 20 & $4(100 \%)$ \\
\hline Suprafascial & 6 & 30 & $5(83 \%)$ \\
\hline Superthin (Periscarpal) & 10 & 50 & $9(90 \%)$ \\
\hline Bone union achieved across fracture line & $(8 / 9)$ & $89 \%$ & \\
\hline External fixator exchanged for internal hardware or removed & $(2 / 2)$ & $100 \%$ & \\
\hline Amputation & 2 & 10 & \\
\hline Radiographically healed compared to preoperative & $(7 / 9)$ & $78 \%$ & \\
\hline Osteomyelitis recurrence & 2 & $10 \%$ & \\
\hline
\end{tabular}

${ }^{a}$ Osteomyelitis clearance as defined by lack of local recurrence of boney osteomyelitis, discontinuation of antibiotic, healed soft-tissue envelope, clearance of deep-space infection

In subgroup analysis of our highest risk populations nine diabetic patients had osteomyelitis and three more had critical limb ischemia. Although this is a small sample size, outcomes in this population are mixed: none experienced recurrence of osteomyelitis defined as clinical evidence of bone infection by clinical exam, radiography or tissue pathology within the surgical site; five of our patients were fully weight-bearing in less than four months while three of them never fully ambulated due to conservative management of secondary ulcers in the same extremity, and one patient went on to amputation due to severe peripheral artery disease. Only one flap loss occurred due to extensive arterial thrombosis despite early intervention within the osteomyelitis group [Table 3].

Microvascular tissue transfer in high-risk individuals harboring vascular disease, osteomyelitis and the Charcot foot improves upon outcomes achieved with alternative standard of care pathways. Revascularization alone as demonstrated in the "Bypass versus Angioplasty in Severe Ischemia of the Leg Trial" (BASIL Trial) ${ }^{[23]}$ offers limited salvage rates with shortened up overall amputation-free survival and mortality when compared to revascularization plus wound directed reconstructive surgery. Of 250 patients 
enrolled and randomized with critical limb ischemia to either open or endovascular revascularization at a mean 3.1 year follow-up: the trial showed a 56\% mortality rate, 38\% amputation-free survival and thirty patients and (7\%) living with an amputation. This of course represents a morbid group of individuals meeting particular selection criteria of the trial, many of whom may be precluded from the surgical stress of free tissue transfer. However, when wound-directed therapy with Integra Bilayer Wound Matrix (Integra Lifesciences, Plainsboro NJ) and skin graft was added to a similar group of individuals, Iorio et al ${ }^{[24]}$ improved limb salvage rates. Limb survival was compared across 105 individuals with 121 foot/ankle wounds according to tissue type exposed and presence of high-risk factors: $61 \%$ of those with bone exposure and osteomyelitis were salvaged, $71 \%$ of diabetic wounds were salvaged and $59 \%$ of diabetics with bone involvement avoided amputation. When provided with thin perforator flaps at our center (18/20) 90\% of individuals avoided amputation with osteomyelitis, (21/22) 92\%, of diabetics were salvaged, all of whom were high-risk for amputation, and $89 \%$ (8/9) of individuals with both diabetes and osteomyelitis avoided amputation.

Hong et al. ${ }^{[2]}$ demonstrated a survival benefit over time in 2016 while utilizing the "Angiosome and Supermicrosurgery Concept" principle and techniques for the management of diabetic foot wounds. Hong et al. ${ }^{[2]}$ salvaged $84.9 \%$ of individuals over five-year follow-up. However, Dr. Hong also noted limited success in those individuals requiring preoperative revascularization. During a regression analysis, revascularization was associated with limb-loss independent of other high-risk features similar to our findings. We came to similar findings as Dr. Hong's with regard to limb ischemia requiring revascularization-lending caution to future patient selection.

With regard to osteomyelitis, flap coverage has been widely studied by several groups over the last four decades ${ }^{[25,26]}$. In 1982, Chang and Mathes ${ }^{[10]}$ described 21 patients with chronic osteomyelitis who underwent muscle flap coverage with a success rate of $90 \%$, two patients (10\%) developed recurrent infection postoperatively ${ }^{[11]}$. Then, in 1991 James et al. demonstrated the long term effect of muscle flap coverage in the management of 34 patients with chronic osteomyelitis with $89 \%$ of success rate over a long-term follow up ( $>5$ years, mean 7.4 years). Reconstructive surgeons readily accepted muscle flaps as a standard for management of Gustillo IIIB defects with osteomyelitis in the 1990's. Eventually fasciocutaneous flaps started to make a presence in the early 2000's. Salgado et al. ${ }^{[1]}$ demonstrated in animal model that both muscle and non-muscle flaps provide a viable option for wound coverage of osteomyelitis defects. A recent publication by Hong et al. ${ }^{[3]}$ assessed the efficacy of perforator flaps in the treatment of chronic osteomyelitis in a retrospective study including 120 patients who underwent reconstruction for chronic osteomyelitis of the lower extremity; their flap loss rate was $4.2 \%$ and partial flap loss rate of $8.3 \%$ with remission rate of $91.6 \%$ in one-stage reconstruction. These findings in line with ours in terms of $6.8 \%$ rate of flap loss overall and $5 \%$ rate of partial flap loss in the osteomyelitis group. Hong et al. ${ }^{[2]}$ utilized 30 superficial circumflex iliac (SCIP) flaps, 1 thoracodorsal artery perforator (TDAP), and 41 ALT flaps but did not describe the plane of elevation. With our contribution of 16 superthin (N. 10) and suprafascial (N. 6) ALT's utilized for osteomyelitis, we estimate the number of published reports of utilizing superthin (periscarpal) and suprafascial flaps for the management of osteomyelitis is in the range of 50-100 worldwide to date.

Notable limitations of this study include our small sample size lending to type 1 error in the comparison of moderate-sized groups of patients with low overall complication rates - necessitating larger numbers to strongly power our conclusions over time. We uniformly relied on the ALT flap at our institution for wounds necessitating coverage by free tissue transfer, which assists in limiting selection biases but is of course a unique practice. The unique referral pattern from foot and ankle surgery, podiatry and vascular surgery along with availability to perform free-tissue transfer in this setting may also be difficult to repeat across centers. This study does not include a number of patients managed by local pedicled flaps, skin grafts, dermal substitutes and local tissue rearrangement. Our limited mean follow-up of 13.8 months 
does not fully capture limb salvage rates particularly in individuals with osteomyelitis who can go on to recur after one year and or those with critical limb ischemia whose disease is not reversed by flap coverage. We also have not explored basic laboratory, animal research or clinical pathologic review facets of fasciocutaneous flaps that promote wound healing. Thus we can comment very little the physiologic basis of our findings. This certainly will be an avenue of further pursuit. Further, this nonrandomized single-site study carries potential selection and treatment biases inherent in unique to the surgeon and institutions practices. However, it is our hope that with further multi-institutional participation, presentation and publication, particularly to our podiatry, vascular surgery and orthopedic colleagues that we can expand our practice and move from small scale low-power studies to larger powered research - working toward prospective trial. This will be particularly helpful in those patients requiring revascularization by either open or endovascular means to delineate selection of patients fit for free tissue transfer and the timing of such interventions.

The superthin, suprafascial and subfascial variations of the ALT flap, are reliable, safe and effective options for lower limb salvage surgery in the setting of osteomyelitis, limb preservation and Charcot collapse. Incorporating these flaps widens the reconstructive surgeon's armamentarium to replace like tissue, avoid a muscle-flap donor, improve contour, shoe gear and allow bony healing - translating to healthy weight bearing limbs and restoring ambulation ${ }^{[27]}$. Limb ischemia necessitating revascularization prior to flap reconstruction remains a major risk factor for limb loss, particularly in the patient with concomitant osteomyelitis.

\section{DECLARATIONS}

\section{Authors' contributions}

Study design: Diamond S, Doval AF, Iorio ML

Manuscript preparation: Diamond S, Iorio ML

Data acquisition: Diamond S, Doval AF, Scott B

Data analysis: Scott B

\section{Availability of data and materials}

IRB approved retrospective study based on BIDMC hospital charted data.

\section{Financial support and sponsorship}

None.

\section{Conflicts of interest}

All authors declared that there are no conflicts of interest.

\section{Ethical approval and consent to participate}

IRB approval prior to data acquisition and study design.

\section{Consent for publication}

An informed consent for publication has been taken from the patients.

\section{Copyright}

(c) The Author(s) 2019.

\section{REFERENCES}

1. Salgado CJ, Mardini S, Jamali AA, Ortiz J, Gonzales R, et al. Muscle versus nonmuscle flaps in the reconstruction of chronic osteomyelitis defects. Plast Reconstr Surg 2006;118:1401-11. 
2. Hong JP. Reconstruction of the diabetic foot using the anterolateral thigh perforator flap. Plast Reconstr Surg 2006;117:1599-608.

3. Hong JPJ, Goh TLH, Choi DH, Kim JJ, Suh HS. The efficacy of perforator flaps in the treatment of chronic osteomyelitis. Plast Reconstr Surg 2017;140:179-88.

4. Anthony JP, Mathes SJ, Alpert BS. The muscle flap in the treatment of chronic lower extremity osteomyelitis: results in patients over 5 years after treatment. Plast Reconstr Surg 1991;88:311-8.

5. Sofiadellis F, Liu DS, Webb A, Macgill K, Rozen WM, et al. Fasciocutaneous free flaps are more reliable than muscle free flaps in lower limb trauma reconstruction: experience in a single trauma center. J Reconstr Microsurg 2012;28:333-40.

6. Suh HS, Oh TS, Lee HS, Lee SH, Cho YP, et al. A new approach for reconstruction of diabetic foot wounds using the angiosome and supermicrosurgery concept. Plast Reconstr Surg 2016;138:702e-9.

7. Yazar S, Lin CH, Lin YT, Ulusal AE, Wei FC. Outcome comparison between free muscle and free fasciocutaneous flaps for reconstruction of distal third and ankle traumatic open tibial fractures. Plast Reconstr Surg 2006;117:2468-75; discussion 2476-7.

8. Lazzarini L, Mader JT, Calhoun JH. Osteomyelitis in long bones. J Bone Joint Surg Am 2004;86:2305-18.

9. Schmitt SK. Osteomyelitis. Infect Dis Clin North Am 2017;31:325-38.

10. Chang N, Mathes SJ. Comparison of the effect of bacterial inoculation in musculocutaneous and random-pattern flaps. Plast Reconstr Surg 1982;70:1-10.

11. Paro J, Chiou G, Sen SK. Comparing muscle and fasciocutaneous free flaps in lower extremity reconstruction--does it matter? Ann Plast Surg 2016;76 Suppl 3:S213-5.

12. Saint-Cyr M, Wong C, Schaverien M, Mojallal A, Rohrich RJ. The perforasome theory: vascular anatomy and clinical implications. Plast Reconstr Surg 2009;124:1529-44.

13. Hong JP, Chung IW. The superficial fascia as a new plane of elevation for anterolateral thigh flaps. Ann Plast Surg 2013;70:192-5.

14. Hong JP, Choi DH, Suh H, Mukarramah DA, Tashti T, et al. A new plane of elevation: the superficial fascial plane for perforator flap elevation. J Reconstr Microsurg 2014;30:491-6.

15. Diamond S, Seth AK, Chattha AS, Iorio ML. Outcomes of subfascial, suprafascial, and super-thin anterolateral thigh flaps: tailoring thickness without added morbidity. J Reconstr Microsurg 2018;34:176-84.

16. Seth AK, Iorio ML. Super-thin and suprafascial anterolateral thigh perforator flaps for extremity reconstruction. J Reconstr Microsurg 2017;33:466-73.

17. Demirtas Y, Kelahmetoglu O, Cifci M, Tayfur V, Demir A, et al. Comparison of free anterolateral thigh flaps and free musclemusculocutaneous flaps in soft tissue reconstruction of lower extremity. Microsurgery 2010;30:24-31.

18. Chen YC. Reply: suprafascial anterolateral thigh flap dissection: limits and advantages. Plast Reconstr Surg 2016.

19. Chen YC, Scaglioni MF, Carrillo Jimenez LE, Yang JC, Huang EY, et al. Suprafascial anterolateral thigh flap harvest: a better way to minimize donor-site morbidity in head and neck reconstruction. Plast Reconstr Surg 2016;138:689-98.

20. Giurato L, Meloni M, Izzo V, Uccioli L. Osteomyelitis in diabetic foot: a comprehensive overview. World J Diabetes 2017;8:135-42.

21. Ruan QZ, Diamond S, Zimmer S, Iorio ML. Assessing the safety and efficacy of regional anesthesia for lower extremity microvascular reconstruction: enhancing recovery. J Reconstr Microsurg 2018;34:293-9.

22. Seth AK, Diamond S, Iorio ML. Outcomes of an early protocol for dependent conditioning in lower extremity microsurgical free flaps. J Reconstr Microsurg 2017;33:670-8.

23. Bradbury AW, Adam DJ, Bell J, Forbes JF, Fowkes FG, et al. Bypass versus angioplasty in severe ischaemia of the leg (BASIL) trial: analysis of amputation free and overall survival by treatment received. J Vasc Surg 2010;51(5 Suppl):18S-31.

24. Iorio ML, Goldstein J, Adams M, Steinberg J, Attinger C. Functional limb salvage in the diabetic patient: the use of a collagen bilayer matrix and risk factors for amputation. Plast Reconstr Surg 2011;127:260-7.

25. Hallock GG. A paradigm shift in flap selection protocols for zones of the lower extremity using perforator flaps. J Reconstr Microsurg 2013;29:233-40.

26. Sisti A, D’Aniello C, Fortezza L, Tassinari J, Cuomo R, et al. Propeller Flaps: a literature review. In Vivo 2016;30:351-73.

27. Maruccia M, Fallico N, Cigna E, Ciudad P, Nicoli F, et al. Suprafascial versus traditional harvesting technique for free antero lateral thigh flap: a case-control study to assess the best functional and aesthetic result in extremity reconstruction. Microsurgery 2017;37:851-7. 